\title{
El diseño y la sustentabilidad social con relación a la aplicabilidad de la noción de objeto
}

\begin{abstract}
Resumen
Este artículo tiene como finalidad indagar, analizar y relacionar, bajo un estudio crítico, la aplicabilidad de la noción objeto, desde teóricos del diseño que pensaron la sustentabilidad social. Cuando Christopher Alexander usa el concepto objeto desde la arquitectura, como un sistema natural o artificial provisto de muchas funciones; Abraham Moles desde la ciencia y la sociología del diseño, como una intención o fin; y Gui Bonsiepe desde el diseño industrial y gráfico, como una interfaz pensada para el usuario, ellos abren sus significaciones a diversas interpretaciones que bien valdrían un análisis particular. En este sentido, una investigación sobre el uso de la noción objeto bajo la concepción de estos autores, permitirá explorar y analizar sus ejes liminares, distintivos y complementarios. Así, se propone un corpus teórico para revisar las formulaciones que ya se han elaborado de la noción de objeto y reflexionar, desde nuevos cuestionamientos, lo que aún se encuentra velado y lo que falta por decir.
\end{abstract}

\author{
Mark Michael Betts Alvear. \\ $\mathrm{PhD}(\mathrm{c})$ \\ Doctorando en Diseño \\ Magíster en Diseño Comunicacional \\ Fundación Universidad del Norte \\ Correo electrónico: bettsm@uninorte. \\ edu.co \\ markmichaelbetts@gmail.com \\ (1) orcid.org/0000-0002-0656-6334 \\ Google Scholar
}

Recibido: abril 25 de 2018 Aprobado: septiembre 11 de 2019

Palabras clave:

diseño, interfase, objeto, sistema, sustentabilidad. 


\section{Design and social sustainability in relation to the applicability of the notion of object}

\begin{abstract}
The purpose of this article is to look into, analyze and relate, under a critical study, the applicability of the notion of object from Design theorists who thought about social sustainability. When Christopher Alexander uses the concept object from architecture as a natural or artificial system provided with many functions, Abraham Moles uses it from science and sociology of design as an intention or purpose and Gui Bonsiepe uses it from industrial and graphic design as an interface rationalized for the user. They open its meaning to diverse interpretations that would be worth a particular analysis. In this sense, research on the use of the notion of object under the interpretation of these authors, will allow exploring and analyzing its liminal, distinctive and complementary axes. Therefore, a theoretical corpus is proposed to review the formulations that have already been developed around the concept of object and to reflect from new questionings, on what is still veiled and what remains to be said.
\end{abstract}

Key words:

Design, interface, object, sustainability, system. 
El arquitecto y matemático Christopher Alexander (2010), en su texto Ensayo sobre la Síntesis de la Forma explica cómo la forma representa el objeto final del diseño, al constituirse para la disciplina como la parte del mundo que se encuentra bajo su control. Tal objeto final implica el alcance de un ajuste eficaz, al proyectar la forma como solución, y el contexto como todo aquello que le exige a la forma. Alexander define como 'ajuste eficaz', a la relación de mutua aceptabilidad entre estos dos aspectos, es decir, entre forma y contexto. Para ello, exalta desde la arquitectura moderna, dos edificaciones muy conocidas como la Casa Farnsworth y Villa Savoye, diseñadas en el mismo orden por Ludwig Miës Van der Rohe, y Charles Jeanneret Gris alias Le Corbusier. Estas casas, las califica como - maravillosamente nítidas -, en razón de que sus diseñadores le apostaron a un orden funcional, que condujo a la minimización de desajustes, es decir, al ajuste eficaz.

La forma es una parte del mundo que está a nuestro control, y que decidimos modelar en tanto que dejamos el resto del mundo tal cual es. El contexto es la parte del mundo que le hace exigencias a esta forma; todo lo que el mundo le hace exigencias a la forma, es contexto. El ajuste es una relación de mutua aceptabilidad entre estos dos elementos. (Alexander, 2010, p. 24)

Alexander se pregunta: ¿por qué a los arquitectos de la actualidad se les escapa de las manos la nitidez que la arquitectura moderna alcanzó en el pasado? Así, critica que esto se debe a tres aspectos: la irresponsabilidad del diseñador frente a las exigencias del contexto, justificada por sus habilidades artísticas especiales, los intereses económicos de quienes encargan los proyectos de diseño y la inadecuada representación ${ }^{1}$ mental del contexto. Alexander sostiene que, si en la arquitectura los diseñadores tomaran en cuenta todos los factores que el contexto le exige a la forma como la ventilación, la variabilidad

\footnotetext{
${ }^{1}$ Para Alexander, el concepto de representación evoca la manera en la que el diseñador establece un dibujo mental del problema u oportunidad que decide abordar desde el diseño. Este dibujo implica también, la relación sistemática entre los requerimientos, sus dependencias y el estricto orden de sucesión y precedencia entre cada una de ellas.
} 
de las temperaturas producto de las estaciones, la circulación del viento y la orientación hacia la luz, etc., entre otros; se alcanzaría siempre el ajuste ideal. Caso contrario, muchos de estos requerimientos son ignorados, lo que facilita el trabajo gracias a la proliferación de artefactos periféricos, que prometen compensar cada una de estas exigencias desatendidas. Si la vivienda carece de ventilación por una incorrecta orientación, si tiene paredes delgadas para responder a la economía y ventanas grandes gracias a un estilo vanguardista, se enmiendan con el aire acondicionado para regular el calor en verano, el calefactor para el frío en invierno, aislantes acústicos y grandes persianas para preservar la intimidad. Sin embargo, no se malinterprete a Alexander, debido a que para él la irresponsabilidad no recae solo en el arquitecto, porque no todos juegan como estetas mal comprendidos, en razón de su individualidad creativa. También se ha de considerar que, la indiferencia al contexto proviene de la plusvalía que los propietarios de los terrenos y los productores financieros buscan extraer detrás de cada proyecto. Al condensar la mayor cantidad de viviendas en un espacio, los sponsor evaden la opinión de los diseñadores frente a la administración correcta del terreno. Estas situaciones no solo limitan las decisiones que el diseñador debe tomar, sino también estimula una incontinencia de los recursos energéticos.

Así en vez de orientar con cuidado la casa en relación con el sol y los vientos, el constructor concibe su organización sin prestar atención a la orientación, y de la luz, el calor y la ventilación se harán cargo ventiladores, lámparas y otros artefactos periféricos. (Alexander, 2010, p. 34)

No obstante, para aquellos escenarios donde se toma en cuenta dicha relación, la solución emerge a partir de la construcción de un esquema del problema, concebido en subsistemas, donde la función de cada uno de ellos no puede entorpecer a los otros que le son liminares en el proceso de diseño. Alexander, consciente de la labor cada vez más compleja a la que se enfrenta el diseñador, identifica cuáles podrían ser los factores que lo alejan del ajuste eficaz, debido 
a que los cambios cada vez más acelerados de las culturas urbanas ${ }^{2}$ conllevan a que sea mucho más difícil conseguir la minimización de desajustes.

\section{Culturas inconscientes y conscientes de sí mismas}

Las posibilidades de alcanzar el ajuste eficaz pueden entreverse en la diferencia entre lo que Alexander denomina como culturas inconscientes de sí mismas y las culturas conscientes de sí mismas. Las culturas inconscientes de sí mismas son aquellas que desarrollan formas a partir de la repetición de pequeños ajustes y cambios devenidos de generación en generación. Ellas edifican bajo pautas establecidas en el pasado, por lo que sus adecuaciones se reflejan en pequeños cambios, a través de los años, de paulatina adaptación; como la única manera correcta de diseñar. La sensibilidad hacia los objetos naturales que el entorno les ofrece, y que sirven como materiales de construcción, son respetados por sus propiedades espirituales. Por eso, sus edificaciones trascienden, debido a que se encuentran influenciadas por tradiciones que hallan su fundamento en sus mitos y cosmogonías. Las culturas inconscientes de sí mismas se ven influenciadas por un tipo especial de inercia contextual: lo que conduce a que sus diseños siempre alcancen el ajuste eficaz. Esta realidad no es la misma para las culturas conscientes de sí mismas. Este fenómeno no se presenta en ellas, porque los cambios culturales constantes afectan los contextos y la forma que buscan solucionarlos. En este tipo de culturas los cambios en sus modos de hacer, pensar y actuar se dan con mayor velocidad frente a la respuesta que los diseñadores pueden desarrollar. Debido a eso, son más susceptibles a los desajustes en todos los subsistemas cada vez más confusos que componen sus edificaciones, productos, servicios y experiencias. Así, el reto para el diseñador se hace cada vez más complejo y "sus posibilidades de éxito son escasas porque el número de factores que debe quedar simultáneamente en su lugar es tan enorme" (p. 63).

Referido a las comunidades que habitan e interactúan en las ciudades, opuestas a las comunidades rurales, que viven en zonas con meno grado de intervención del hombre en relación con el ambiente natural. 
El diseñador, al percatarse de esta responsabilidad, hace un esfuerzo por tratar de copiar la manera inconsciente, para que, en el proceso de minimización de desajustes, sea mucho más fácil superar los requerimientos. Pero el problema yace en que cada subsistema que busca estructurar, cumple con una función que se desactualiza permanentemente, por la velocidad con la que cambian sus contextos. En la búsqueda del ajuste eficaz, el diseñador utiliza sus conocimientos y experiencia para crear una representación mental del contexto, pero al no tener un control absoluto de la cantidad de variables, la representación es errada. La solución que propone Alexander consiste en abstraer el proceso de representación mental que el diseñador tiene y adecuarla a otra representación matemática. En la que la imagen mental del problema se reconstruye a partir de pequeñas unidades, que permiten tener control sobre la cantidad de desajustes, porque no están limitados por variables cuantificables. La representación matemática del contexto en unidades de conjuntos y subconjuntos, le ofrece al diseñador una comprensión total del problema, gracias a la identificación de cada una de las variables que se encuentran inmersas en él. Así, se logran superar con claridad los desajustes, a partir de la mutua aceptabilidad que comprende la forma y su contexto. En palabras de Alexander, el objetivo final del diseñador es la forma, aunque "cuando hablamos de diseño, el objeto real de la discusión no es sólo la forma, sino el conjunto que comprende la forma y su contexto" (p. 22) (figura 1). 


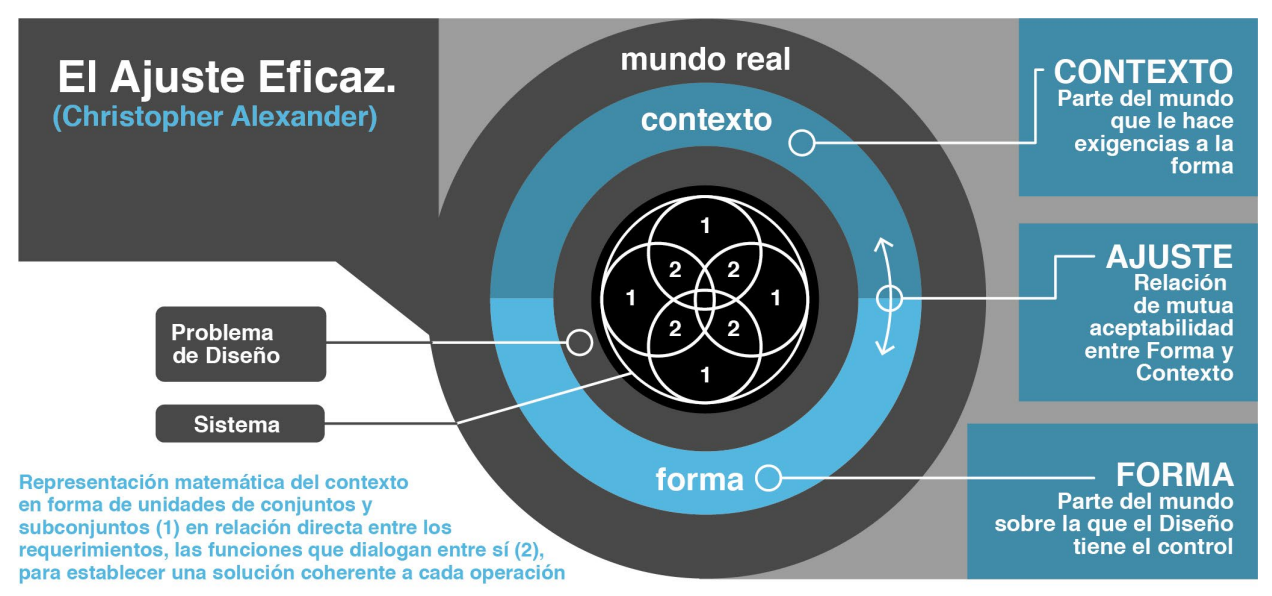

Figura 1. El ajuste eficaz, para Christopher Alexander. Fuente: Elaboración propia del autor.

De todas formas, en Alexander, la manera de representar los contextos que distingue las culturas inconscientes de sí mismas, de aquellas conscientes de sí mismas, nos recuerda lo que el diseñador austriaco Víctor Papanek (1984), en su texto Design for the real World, reconoce como la capacidad potencial de diseñar que poseen todos los hombres, y que es constitucional del pensamiento proyectual. Para Papanek, el pensamiento proyectual aparece como un acto planificador hacia un fin deseado, un diálogo entre un método de ordenamiento innato y otro especializado en constante cambio; liminar a la representación matemática del contexto de Alexander. Papanek deja entrever que no son los grados de especialización del método los que separan la intención real y espontánea del acto de diseñar que encarna la esencia de lo proyectual detrás de cada objeto, sino el intento de autonomizar al diseño con fin en sí mismo, en contra del valor inherente por el cual surge. Facilitar la calidad de vida de todas las personas a través de los procesos de la investigación y la creación define la función primordial del diseño, sin olvidar que los diseñadores, en su 
entorno laboral, dependen de las dinámicas de un sistema económico. Si bien este interés, representa la finalidad del diseño, tanto para Alexander como para Papanek, existen intenciones de carácter político sujetas al motor económico e industrial que no pueden obviarse, y que más allá de las funciones prácticas, se instauran en las dinámicas que unen a los diseñadores, con la producción y el consumo. Dicho carácter político, y se aclara que no siempre, al determinar los hábitos y las conductas de interacción entre las personas y sus objetos, en relación distintiva con otros objetos, funcionan como mediadores culturales poco afirmativos; dentro del entorno natural y artificial que rodea al hombre. Y son realmente estos condicionamientos, los que hacen que el modelo de representación de las culturas conscientes de sí mismas, se alejen cada vez más del ajuste ideal, y al diseño de su finalidad real.

\section{La praxis cultural, artificio condicionante}

Si bien el concepto de contexto, Alexander lo usa para incluir allí las exigencias que el ambiente natural y artificial le exigen a la forma, el entorno natural como lo conocía el hombre del siglo XIX, en su contacto afectivo, es posible que en la actualidad no haya desaparecido. Dentro de los espacios en los que el hombre contemporáneo interactúa, se encuentra condicionado socialmente por leyes de carácter político, gracias a la apariencia artificial, material y abstracta de sus mediadores. Dichos mediadores se encuentran para el teórico del diseño Abraham Moles (1990), según su libro El Kitsch, en la forma y el contenido de cada objeto que lo rodea; debido a que influyen en la construcción de los valores, las actitudes y los estilos de vida de quienes los adquieren. De allí que Moles considere lícito denominar a la cultura al "ambiente artificial que se creó el hombre por medio del cuerpo social" (p. 12), donde los objetos como sus mediadores condicionan constantemente, la naturaleza de cada una de sus relaciones. Los objetos para Moles no son las cosas de uso cotidiano, las cosas representan todo lo que hace parte del ambiente natural, pero que 
al ser artificializadas se convierten en objetos. "Una piedra es una cosa, y se transformará en objeto cuando se la promueva a la dignidad de pisapapeles mediante la industria humana del souvenir y lleve una etiqueta: 'Precio.: ... Calidad: ..."'. (p. 34). Es decir, cuando se les inocula la impronta y la expresión misma de la sociedad; debido a eso, los objetos se transforman en agentes del entorno material de la sociedad tecnológica, y por ende en constructores de cultura.

\section{Una dialéctica entres situaciones, actos y objetos}

Para que estos mediadores puedan desempeñar sus papeles edificadores, una dialéctica fundamentada entre situaciones, actos y objetos, debe ponerse en práctica. Tal dialéctica se encuentra lubricada por coeficientes cuantificables, o valores de atracción que articulan a los hombres, los objetos y un sistema de consumo estructural, en una relación que tiene como fin la sustentabilidad económica del mundo en el que se - debe- prosperar. En este nuevo mundo, las situaciones representan eventos culturales cotidianos, como el cumpleaños de un familiar, la inauguración de un shopping mall, las actividades de diversión y esparcimiento o una cita con una posible pareja, etc. Estas situaciones alisan el terreno donde se condicionarán, cada una de sus necesidades y donde sus actos representan las tareas que debe realizar para solucionarlas; espacio artificial de su praxis cultural. Se compra un obsequio para materializar una transacción de sentimientos, se va de vacaciones para relajarse, y se rocía el último perfume francés lanzado al mercado para atraer a alguien que nos gusta. Para Ilevar a cabo todos estos actos, los objetos se hacen necesarios, y se hacen indispensables desde el momento en que se justifican bajo la función y la utilidad. Así, lo ratifica Moles: "las situaciones generan, en los individuos, actos para salir de esas situaciones. En esos actos utilizan mediadores, que son los objetos que la sociedad provee al individuo" (p. 51). Esta dialéctica que emerge como una relación dependiente e hipostasiada entre el hombre y sus objetos, se basa en 
una cultura consumidora "que produce para consumir y crea para producir, en un ciclo cultural cuya idea fundamental es la aceleración" (p. 22) (figura 2).

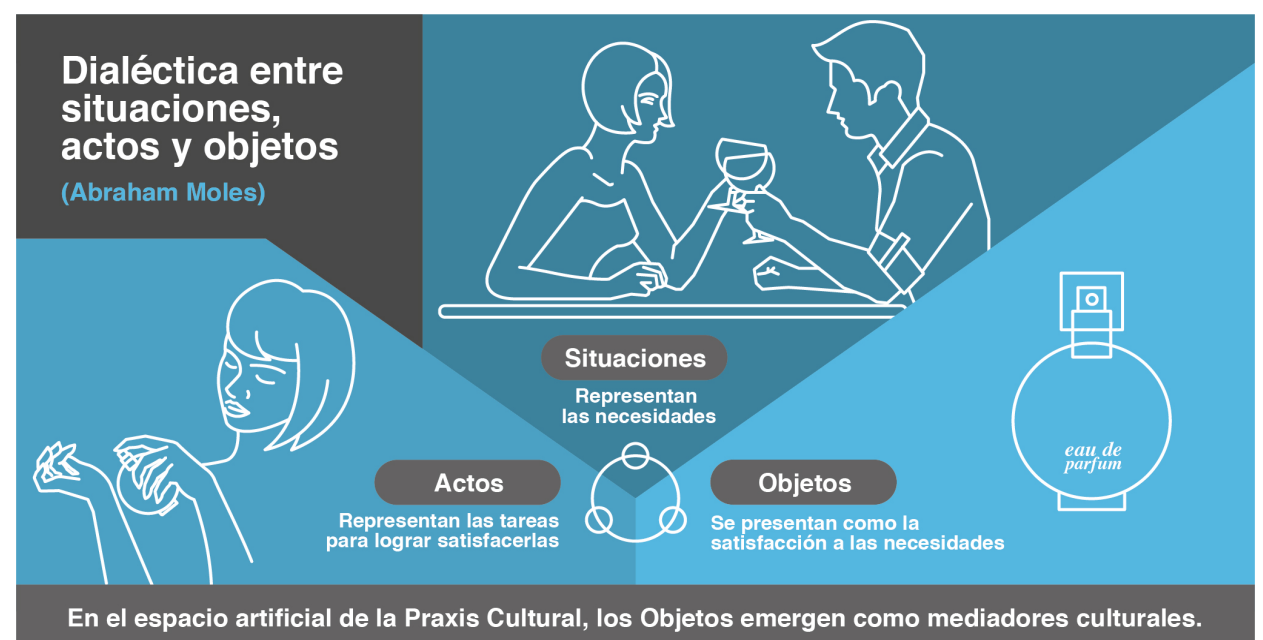

Figura 2. Dialéctica entre situaciones, actos y objetos, para Abraham Moles. Fuente: Elaboración propia del autor.

Ese mundo se caracteriza por la intervención entre el hombre y la sociedad, de mediadores que lo invaden cada vez más y que transforman la naturaleza misma de sus relaciones. Las relaciones del individuo con el medio social pasan, en lo sucesivo y fundamentalmente, por los objetos y productos, transformados en las expresiones más tangibles de la presencia de la sociedad en su ambiente, desde el momento en que ocupan el lugar de las 'cosas naturales' [...] Estos seres, a su vez, retroceden a un segundo plano, por detrás de la reorganización mecánica de los bienes y los servicios. (Moles, 1990, p. 12).

\section{Dimensiones semiológicas de los vectores atracción}

Sin embargo, para que los objetos puedan operar de esta manera, la dinámica debe vincular elementos de atracción que se manifiestan desde los planos 
semiológicos de la denotación y la connotación. Moles sostiene que los valores como coeficientes numéricos caracterizan a los vectores de atracción en un campo de representación, que se manejan como valores. Uno es el valor de uso, que representa la utilidad práctica o el para qué está hecho un objeto, que cumple o promete una o varias funciones, bajo la instancia denotativa que lo significa: -lavadora-. Dos, el valor de cambio económico que sustenta la lógica primaria del capitalismo y que estudió el economista prusiano Karl Marx. Dicha lógica establece que las personas trabajen para intercambiar sus fuerzas de producción en un tiempo obligado por un equivalente económico, el dinero. Éste se vuelve a intercambiar en sus actividades de tiempo libre, con la adquisición y uso de los objetos. Este ciclo que mueve la economía bajo el consumo y la producción, se sustenta sobre el valor de uso, el valor de cambio y las necesidades. Pero la sociedad que hoy rige al hombre es muy compleja, y ¿cómo se explica qué elementos lo seducen para que elija un objeto en vez de otro? El tercer valor, denominado estético, es el que opera, para Moles, en el plano de las connotaciones, al usar elementos que son manipulables para la alienación del hombre; y es en esta posición donde el hombre no sabe realmente qué es lo que quiere.

El tiempo del trabajo productivo solo aparece, de ahora en adelante, como un preludio, necesario pero provisorio, de la automatización total del proceso [...] y esencialmente, lo encontramos en una inmensa actividad consumidora — teleguiada, como diría Riesman — pero que tiene un carácter personalizado y se sitúa en el extremo opuesto de la acción de producción y del trabajo masivo. (Moles, 1990, pp. 24-25)

Este valor estético inoculado en la dimensión connotativa del objeto alberga en su interior elementos sígnicos sobreañadidos: como la sensualidad que se promete en lo que se desconoce, y la vinculación con la posesión que, concebido bajo el principio de la belleza, persigue con sus signos la diferenciación de su propietario al creerse con la ilusión de poseerla. Adicionalmente, los atributos novedosos de la forma producen fiebre de 
compra en el consumidor, que solo se cura solamente con la adquisición. Todos estos elementos, sobredimensionados por la publicidad (proceso en el que se le atribuye al objeto una imagen idealizada), difunden la promesa de cumplimiento de todos los deseos, desde el ritual de la extracción de la vitrina y/o de los entornos virtuales de compra, hasta la ubicación en los diferentes espacios establecidos como lugares de veneración. Se adora la TV junto a los servicios y las experiencias que generan las imágenes en $4 K^{3}$ en el altar de la habitación principal, los smartphones de última generación en espacios concurridos en relación con el potencial interactivo y distintivo frente al otro, el auto deportivo en la playa en proporción diferencial con otros autos y las copas de Murano en las reuniones con los amigos.

Las aspiraciones motivacionales se manifiestan, de manera inconsciente, gracias a una sustancia velada que reposa en el alma de cada objeto diseñado y proyectado para que el hombre sienta que elige; gracias a este valor estético de las formas. Las aspiraciones se reflejan ahora en el espacio personal del individuo, en los objetos que lo rodean y que proyectan un aura de estatus diferenciadora ante el otro, bajo los signos del ideal de comfort y la belleza, o de la supuesta aproximación a ellos. Un espacio artificial cobijado por sus objetos manufacturados, donde su sensibilidad y percepción nacen atrofiadas. Una sociedad que nutre de cultura a los consumidores a través de sus objetos, como vehículos de sus servicios y experiencias, una sociedad de consumo, llámese mejor, en términos de Moles, una - sociedad objetal- . Los constructores de objetos tienen como responsabilidad el análisis y la conjunción racional entre muchos elementos. El diseñador emerge como su constructor que, comunica en concesión, las funciones dictaminadas por las promesas hechas a cada uno de sus usuarios; dependientes de los requerimientos elaborados desde el mercado. Según Moles, es de esta manera, cómo el diseñador toma las visiones

${ }^{3}$ Resolución de alta definición en dispositivos high tech, como los Smart TVs UHD. Dicha resolución corresponde a: 3840 x 2160 pixeles, con relación widescreen 16:9. 
avanzadas del artista, muy avanzado para su época, y las reacomoda con la capacidad técnica y recursiva de invención de los ingenieros; en un ciclo que administra la capa sociocultural. Esta sociedad objetal le ofrece al individuo una catarsis a sus deseos, en un espacio libre donde los objetos, actúan como reformadores de cultura y también como vehículos para relacionarse (figura 3).

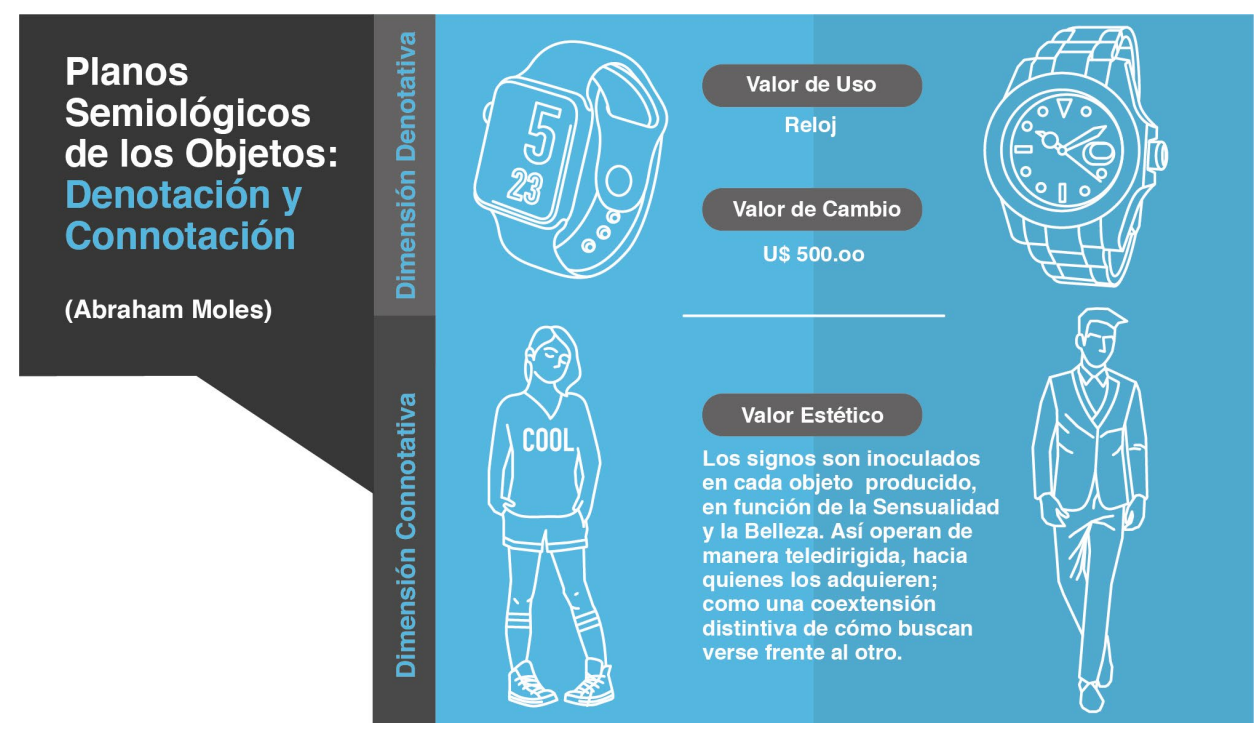

Figura 3. Planos semiológicos de los objetos, para Abraham Moles. Fuente: Elaboración propia del autor.

Subrayemos esta acción del diseñador, situado entre el artista y el ingeniero, que realiza la nueva función del product engineer, la ciencia del arte aplicado, y que rechaza la oposición arte/ciencia y se inserta en la cadena creación/difusión. Toma sus novedades del arte o de la ciencia y, en el plano técnico. (Moles, 1990, p. 187) 
Éticas de la creación o de la destrucción

Pero no se malinterprete a Moles, dicha sociedad objetal no se rige por un simple vector de atracción que conduce a la acumulación. Contrario a ello, una de sus intenciones, en el sistema de consumo actual, consiste paradójicamente en no aumentar la saturación de las posesiones. Así, Moles explica cómo las culturas ancestrales (egipcios, Aztecas, Mayas e Incas, etc.) conscientes de su fragilidad humana, construyeron, con el fin de trascender y perdurar. Sus legados son testigos de una - Ética de la eternidad-. El hombre de esa época se preocupaba por la permanencia de sus obras, tendiendo sus objetos a ese principio como función esencial. En la época industrial esa lógica sufre una transformación que, bajo la ostentación propia de las sociedades agrarias, reinó con la sedimentación acumulativa de los objetos. Un momento en que la emancipación de la cultura burguesa mostraba índices de exceso de los medios comparado con sus necesidades, lo que colocó a los objetos como agentes de su dinámica clasista, gracias a la -Ética de la acumulación-. Ésta, la del fenómeno Kitsch, un modo de vida más que un estilo, en el que a los objetos del siglo XIX se le sumaba una función social sobreañadida a su función semántica, según Moles, necesaria para el amontonamiento del feliz poseedor; materializada bajo la irracionalidad del ornamento, la decoración y el desperdicio de los recursos en su fabricación. "El Kitsch es, una función social sobreañadida a la función significativa de uso, que ya no sirve de soporte sino de pretexto" (p. 27).

En la actualidad, los individuos se encuentran atrapados en un ciclo de la aceleración o - en la Ética de la destrucción creadora - , tal como Moles (1981) sostiene en su texto Théorie des Objets, (p. 108); lugar donde la acumulación de los objetos que configuran su Umwelt ${ }^{4}$, o ambiente, no es la que determina su estatus de clase, sino la velocidad con que los actualiza constantemente. 
Este modo de consumo que apela a la ética de la destrucción creadora recibe su nombre a partir de las implicaciones ecológicas peyorativas, producto de las sociedades ya no del uso, sino de la prodigalidad, sustentada en el reemplazo y el desecho prematuro de los objetos. En otras palabras, es un fenómeno Neokitsch que se redefine por lo perecedero, es decir, por una corta vida diseñada junto a una motivación efímera de posesión; en términos de Moles, es el paso del Kitsch al NeoKitsch. Sin embargo, este deslizamiento del consumo de la época industrial hacia el consumismo desmedido de mensajes y signos de esta sociedad objetal, usa el Bauhaus como excusa, al inocular funcionalidades tangibles o virtuales a cada objeto inútil que se produce. Moles deduce que si en las sociedades tradicionales, el tiempo de caducidad era establecido bajo la indiferencia del consumidor, hoy se inscribe sobre su conciencia, con la finalidad de inducir a la aceleración, bajo las etiquetas comerciales de: garantizado por un año; el objeto es entonces condenado a una prematura destrucción, como nueva forma de civilización.

La caducidad es, pues, una confrontación del objeto y de su ser con el ciclo sociocultural; establece una fecha, no por el desgaste, que actúa generalmente sobre la función semántica del objeto, sino sobre el sistema de valores estilísticos ajenos que se superponen a la funcionalidad. La caducidad inscribe la fecha de compra, en la evolución del entorno social. (Moles, 1981, p. 102)

Los objetos exhiben en su materialidad/virtualidad y en su velocidad de recambio la significación del estatus, como el nuevo imaginario social de prestigio distintivo, producto de las aspiraciones que se excusan sobre las necesidades prácticas. Son entonces por prioridad, el recurso preponderante de una - sociedad objetal-, los índices del ser y de la clase social de su poseedor y de sus aspiraciones. El factor connotativo de los objetos que se engendra desde el valor estético, no puede darse de forma aislada, necesitará siempre de la denotación, área propia de la funcionalidad, aunque la segunda le sirva muchas veces como excusa. 


\section{Remanente biológico}

No se malentienda a Moles: los reflejos cada vez más extintos de las necesidades biológicas se ven limitados por las adaptaciones complejas propuestas por la cultura. Así, si el hombre de las cuevas de Altamira saciaba su sed bebiendo del río, el contemporáneo lo hace también, pero desde la infinidad de bebidas que ha creado y que se ofrecen desde el mercado, condicionado de manera tipológica por nuevas reglas culturales que involucran toda una taxonomía de objetos, formas y experiencias. Se bebe agua en botellas plásticas en un triatlón, cerveza en lata con los amigos en una fiesta y se aprecia la cepa del merlot en copas de cristal en un aniversario. La utilidad práctica desde la denotación prometerá vanamente satisfacer una mayor cantidad de condicionamientos culturales a las necesidades, a través de una vertiginosa cantidad diversa de objetos. Así, las motivaciones sociales en forma de signos, impulsados por las nuevas figuras del diseño de servicios y los ideales de superación, continuarán moviéndose. Todo ello, con el objetivo de atraer y sumergir al hombre en una actividad adquisitiva permanente, gracias al plano dinámico de las connotaciones. Es de esta manera, cómo los objetos, en su papel co-extensivo de los actos humanos, invitan a ser partícipes de esta nueva vida socializada; lugar donde el Umwelt, se encuentra cada vez más artificializado.

\section{Esquema ontológico del diseño}

Si bien los objetos, para Moles, son mediadores de la cultura, la sociedad objetal, por la que el diseño supuestamente trabaja, hace del Umwelt un entorno cada vez más artificial. Sin embargo, la operatividad del diseño no podría limitarse solo a un campo laboral donde cada uno de sus profesionales se debate entre el deber de crear objetos útiles o inútiles, en razón de una demanda de productos y servicios motivada solo en beneficio de la economía. El concepto objeto se encuentra sujeto también a una función social que, 
ontológicamente hablando, los intereses del mercado no podrían aparecer como su única motivación.

Las mercaderías, tal como las denomina el teórico alemán del diseño Gui Bonsiepe (1999), en su texto Del objeto a la interfase, engloba toda esa categoría de gadgets de las que tanto habla Moles, y que no pueden representar la totalidad de la práctica del diseño. A través de lo que Bonsiepe denomina como el esquema "ontológico del diseño" (p.17), el concern emerge como los requerimientos de un usuario o agente social que se encuentra frente a un problema. El concern consiste en la primera preocupación del diseñador, pues las necesidades aparecen como su primer aspecto a resolver. Estas dependen de diversas tareas, que se deben solventar para satisfacerlas; lugar donde el utensilio si funciona como una coextensión humana. Así, si alguien (usuario) necesita - colgar - un cuadro en la pared (tarea o acción) elegirá un martillo (utensilio), para lograrlo. Estos tres elementos: - usuario, tarea y utensilio - se conectan a través de un espacio de interacción denominado interfase ${ }^{5}$, área instrumental del diseño industrial y del diseño gráfico. "La interfase vuelve accesible el carácter instrumental de los objetos y el contenido comunicativo de la información. Transforma los objetos en productos; transforma la simple existencia física (Vorhandenheit), en el sentido de Heidegger, en disponibilidad (Zuhandenheit)" (pp.17-18). Por ejemplo: el pocillo para beber está compuesto por un recipiente cilíndrico y una oreja. El recipiente (01) servirá para soportar el líquido frío o cálido por determinado tiempo. La oreja (02) y el borde (03) estarán condicionados ergonómicamente para no maltratar los dedos mientras se sostiene con las manos, y la boca a su borde circular, sin riesgo de quemarse o maltratar al beber. La oreja soldada al envase y las superficies romas son los elementos que dan su razón de ser al utensilio como pocillo, es decir, en tanto producto. La interfase es el intermediario o el espacio instrumental que establece una relación objetiva y funcional entre el utensilio y el usuario; es decir la intención final del diseño, entre varias para Bonsiepe (figura 4). 


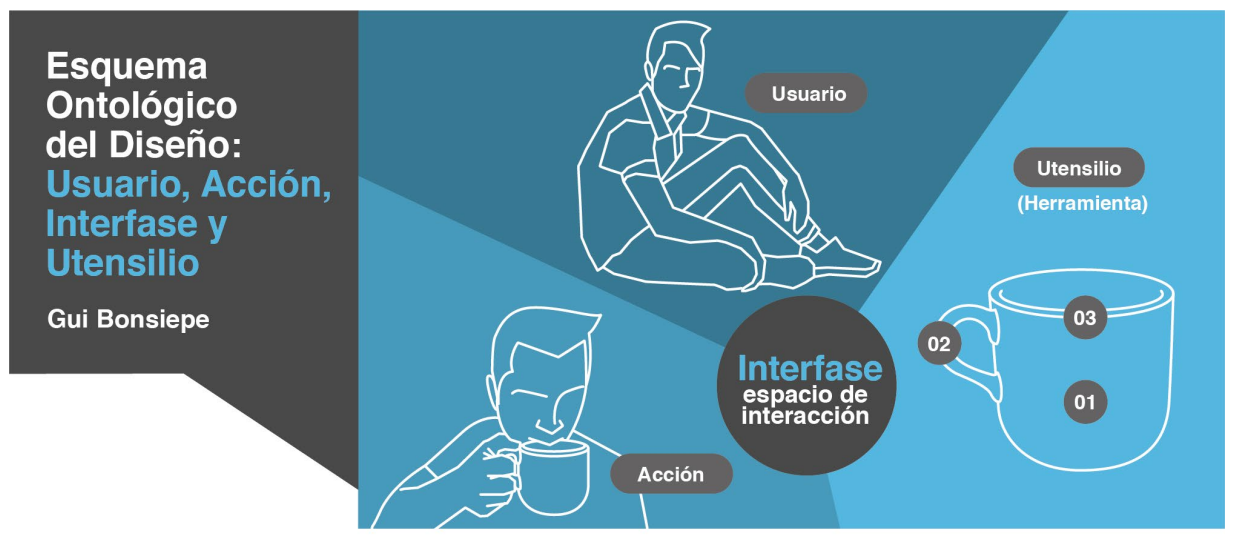

Figura 4. Esquema ontológico del diseño, para Gui Bonsiepe. Fuente: Elaboración propia del autor.

Los cálculos y las consideraciones que se toman en cuenta en la proyectación de los productos en el diseño, como constructor de la cultura material humana, junto a todas sus intenciones comunicativas, evidencian que el trabajo de los diseñadores dista mucho de la simple producción de mercaderías, gadgets efímeros y el lip service que parecen caracterizarlos. Estos recursos, que se originaron para Bonsiepe en los años treinta del siglo XX, continúan influyendo sobre el discurso del diseño de la actualidad, provenientes de una aberración conocida como el Styling Design, impulsada por diseñadores como Raymond Loewy, Henry Dreyfuss y Norman Bel Geddes, entre otros.

Hiperconsumo, subconsumo y mercaderías

Pero, ¿qué es para Bonsiepe el lip service y el styling design? La proyectación de la forma de los objetos en la época del styling se enfocó menos en las funciones técnicas, y más en las funciones estéticas, para crear propuestas que 
no alcanzaron la calidad requerida del producto. El styling es para Bonsiepe (1985) un simulacro, tal como lo sugiere en su libro El diseño de la periferia, que, al operar solo en una parte de las funciones, aparentó una actividad integral de la práctica del diseño, lo que derivó en una tendencia hacia el "hiperconsumo y complicidad con el sistema de intercambio desigual" (p. 22). El hiperconsumo, que motiva a las personas a comprar cosas que no necesitan cada vez con mayor velocidad, apareció inicialmente en Estados Unidos como una reacción a la sustentabilidad económica, posterior a la depresión de los años treinta del siglo XX. El sistema capitalista que lo puso en práctica le otorgó a este fenómeno, en los contextos de los países de la zona centro, un lugar preponderante como la base de su imperativo de mercado; que más tarde les exigiría a los diseñadores, adecuarlo en forma de colonialismo cultural hacia la periferia.

\section{Consumo, hiperconsumo y subconsumo}

Se entiende como "consumo" a aquella actividad que satisface las necesidades de una sociedad a partir de la adquisición de productos, con base en exigencias prácticas y simbólicas demandadas por las personas. El consumo, como fenómeno social, resuelve a través del diseño, las necesidades. Sean los objetos tangibles o intangibles que emergen de cada una de las fronteras del diseño, como el arquitectónico, de productos, de indumentaria, gráfico, etc., entre otros, no pueden concebirse hoy sin una vinculación con el diseño de experiencias. De allí que los diseñadores al condicionar las formas de relacionarse con el otro, usen de intermediarios a los objetos; por ello son visualizados como mediatizadores de la cultura. No obstante, si bien es claro que toda sociedad avanza tomando en cuenta los medios tecnológicos de su época y las relaciones de producción como mecanismos de administración social de sus recursos, para Bonsiepe, el consumo ha desarrollado dos patologías que desdibujan el esquema ontológico del diseño. 


\section{Centro y periferia}

A través de la historia, el registro de la cultura, junto al inventario de todos sus objetos, producto de la evolución social del hombre, ha venido sufriendo cambios. La tecnología de una época es prueba de ello, debido a que condiciona de forma cuantitativa y cualitativa todos los productos que cada generación desarrolla. Este nivel de desarrollo no está repartido ni instrumentalizado en todos los países por igual. Existe una diferencia en cómo se manifiesta la cultura material: el sistema de los objetos concebidos y proyectados en los países de la zona centro y los proyectados en la periferia. Si bien el diseñador funciona como una especie de "físico de la cultura" (p. 16), es el que mediatiza con poder e influencia en este sistema de los objetos. Pero este sistema no depende tanto del diseño como sí del consumo, que determina bajo una relación vertical las relaciones desde la zona centro hacia la periferia. Los diseñadores, de la periferia, en vez de proyectar objetos que satisfagan las necesidades objetivamente, alienados por la imitación de las exigencias de la zona centro, asimilaron todos sus valores como propios en un papel de subalternos:

Por cierto, no faltan las cajas de resonancia, es decir, las tendencias locales de la periferia que reproducen y emulan in situ el diseño industrial, tendencias del mimetismo cultural, ansiosas por recibir el espaldarazo oficial del centro. Nos encontramos así ante una de las más tristes, aunque comprensibles formas de alienación: la pérdida de la propia existencia [...] fenómeno que no solamente puede agobiar a los proyectistas, sino también y en menor grado, a industriales y políticos de diseño de la periferia. (Bonsiepe, 1985, p. 18)

\section{Producción vs. comercialización}

El diseño industrial en los países desarrollados se gestó como un mecanismo de solución a los requerimientos prácticos de su espacio, solucionándolos a partir de la adecuación de sus avances y la producción de formas para la masificación. La realidad en la periferia fue diferente. La desventaja tecnológica entre países 
desarrollados versus en desarrollo, llevó a que el diseño ingresara a los países periféricos, como los de Latinoamérica, con la reproducción de lo que se hacía en el centro, con estatutos de difusión y comercialización. De esta manera era inevitable que el diseño respondiera inmediatamente con el desarrollo de un sistema objetivo de producción. Las únicas áreas que quedaron bajo el dominio de la periferia fueron el marketing y la publicidad, al cederle al centro la creación y la masificación.

\section{El make up y el lip service}

Una saturación de las lógicas del mercado de los países centrales, manifestaron un fenómeno de proliferación de necesidades, que se prometieron satisfacer con una superproducción mayor de objetos. Los remanentes retornaban muchas veces hacia la periferia, lo que generó una codependencia sin resistencia. La periferia no solo absorbió el excedente de la productividad importada, sino que su producción, o se debería decir, su - reproducción-, imitó a la zona centro. Es decir, el avance de los tecnólogos y diseñadores de los países del centro se convirtió en un modelo a imitar, sin considerar la relación coherente que debía existir entre contexto, técnica, forma e interacción con el usuario, lo que obvió el esquema ontológico del que Bonsiepe habla. No parecería raro que el make up y el lip service fueran acogidas por los diseñadores de la periferia, donde Bonsiepe, al recordar las críticas del diseñador suizo Max Bill, señala dicho fenómeno como la cosmética del discurso proyectual (Bonsiepe, 1999, p. 17). Así, las mercaderías como remanente importado, fueron comercializadas gracias a los medios de difusión. La modalidad desmedida de producción y adquisición del centro, junto a la comercialización de sus remanentes y las imitaciones maquilladas por la periferia, representaron una desfiguración del consumo que debió sustentar otra clase de objetos. 
El subconsumo es represivo tal como el hiperconsumo es anestesiante. Ambos fenómenos indican que algo anda mal en este mundo [...] si se crea en la periferia la tecnología y el diseño, pues es ésta la única manera de romper el círculo vicioso de la dependencia tecnológica. (Bonsiepe, 1985, p. 22)

Según Bonsiepe, el diseñador como físico de la cultura promete, a partir de la creación de sus objetos tangibles e intangibles, una relación objetiva entre los usuarios y el consumo, éste último como el espacio social donde interactúan y los adquieren. No obstante, la promesa a la satisfacción de las necesidades, se vio desdibujada por el hiperconsumo de los países de la zona centro y el subconsumo originado en la periferia.

\section{Conclusiones}

Un estudio del concepto objeto desde la praxiología humana, como lo propone Moles, evidencia los aspectos con los que se enviste el sistema económico y los fenómenos ligados a él, que operan desde dimensiones semiológicas. La denotación como una especie de conciencia política, justifica cada objeto diseñado como la satisfacción de las necesidades. Y la connotación, impulsada por el consumo desmedido de objetos, mensajes y signos genera una cultura que produce para consumir y crea para producir. Moles afirma que todo lo que pertenece al ambiente natural se les denomina cosas, pero que trascienden a la categoría de objeto solo cuando se las promueve por la industria humana. De lo que se deduce que, el hombre como un producto de la naturaleza, se transforma en objeto cuando es subordinado por la reorganización mecánica de los bienes y los servicios a la que pertenece y no al revés. Si la sociedad objetal subsiste en un espacio cada vez más artificial, el consumismo visiona, tanto en Moles como en Bonsiepe, una imagen cercana del hombre asfixiado por mercaderías y gadgets desechados. Si bien es cierto que el diseño trabaja para la producción de mercaderías, también lo hace para la reconstrucción de un nuevo mundo; donde funciona en beneficio de la sociedad y no para unos pocos. Bonsiepe protesta contra el juicio que se le impugna al Diseño 
y a los diseñadores como meros productores de mercaderías, como si ese simulacro representara la práctica del diseño en su totalidad. Así, critica el confundirlo con el styling, ya que constituye una herramienta sin objetivos sociales, conducida por una compleja racionalidad de mercado. No obstante, se deduce que tampoco se puede sostener que toda la práctica del diseño en función del mercado puede considerarse peyorativa, debido a que existen líneas de productos lifetime warranty, creados con objetivos más universales que utilitaristas. Bonsiepe, al aclarar la diferencia entre consumo, hiperconsumo, subconsumo, deja clara la segmentación con la que se le acusa al diseño. Y aunque su esquema ontológico guarda cierta relación con la dialéctica entre situaciones, actos y objetos, propuesta por Moles, la segunda se concentra en la mediación cultural que los objetos logran sobre el ser humano en relación con su entorno. Por otra parte, el esquema ontológico se enfoca en la instrumentalidad del objeto humanamente utilizable, gracias al espacio de interacción que genera la interfase. Dicho esquema propende por la creación de objetos para una aplicación organizada del consumo, y no para el consumismo. El transformar la existencia física en disponibilidad, propuesta por Bonsiepe, vendría a representar para Alexander el objeto final del diseño, debido a que su preocupación se concentra en la mutua aceptabilidad entre la forma y el contexto. El interés de Alexander radica en cómo la praxis del diseño, a partir de un proceso de sistematización, conduce a la minimización de desajustes; ya que la forma es la parte del mundo que la disciplina tiene a su control. Sin embargo, también considera que todo lo creado por la naturaleza y por el hombre es un objeto, siempre y cuando, éste último, alcance siempre y en cada una de sus propuestas el ajuste eficaz ${ }^{6}$.

${ }^{6}$ Este texto corresponde a la síntesis de la tesina realizada para la obtención del título de Especialista en Diseño Comunicacional en el DiCom, FADU. U.B.A. Universidad de Buenos Aires. 


\section{Referencias}

Alexander, C. (2010). Ensayo sobre la síntesis de la forma. Buenos Aires: Infinito.

Bonsiepe, G. (1999). Del objeto a la Interfase. Mutaciones del Diseño. Barcelona: G.G.

Bonsiepe, G. (1985). El diseño de la periferia. Barcelona: G.G.

Marx, K. (1980). Contribución a la crítica de la Economía Política. México: Siglo XXI.

Marx, K. (2008). El Capital. Crítica de la Economía Política. El Proceso de Producción del Capital I. México: Siglo XXI.

Moles, A. (1990). El Kitsch. El arte de la felicidad. Buenos Aires: Paidós.

Moles, A. (1981). Théorie des Objets. Sao Paulo: Biblioteca Tempo Universitario.

Papanek, V. (1984). Design for the Real World. Chicago: Academy Chicago Publishers.

Como citar: Betts, M.M. (2020). El diseño y la sustentabilidad social con relación a la aplicabilidad de la noción de objeto. Revista KEPES, 17 (21), 61-84. DOI: 10.17151/kepes.2020.17.21.3 